\title{
Mind the gap - using clinical audit to minimise medication information errors at hospital discharge
}

\author{
Ashok Kumar Jainer, ${ }^{1}$ Fabida Noushad, ${ }^{2}$ Tim Coupe, ${ }_{1}^{1}$ Chaya Rekha Mupiri, ${ }^{3}$ Anoop Saraf ${ }^{4}$
}

The Psychiatrist (2010), 34, 248-250, doi: 10.1192/pb.bp.109.026963

${ }^{1}$ Coventry and Warwickshire Partnership NHS Trust; ${ }^{2}$ Leicestershire Partnership NHS Trust: ${ }^{3}$ Central and North West London NHS Foundation Trust; ${ }^{4}$ South London and Maudsley NHS Foundation Trust

Correspondence to Fabida Noushad (fabida@gmail.com)

\begin{abstract}
Aims and method We conducted a retrospective audit of 100 discharge summaries to evaluate the accuracy of medication recording and the recording of as required (PRN) prescribing, and to see whether or not general practitioners were advised on how long to continue the latter. After a formal guideline was introduced we conducted a re-audit.
\end{abstract}

Results There was an improvement in summaries recording medication correctly (from 64 to $83 \%$ ). The number of summaries with one or more missing medications halved and PRN sedative prescribing reduced from 18 to $3 \%$, but provision of advice on the latter did not improve.

\begin{abstract}
Clinical implications Accurate recording of medication in the discharge summary is an important element of the transfer of patient care to the general practitioner. Medication errors may pose serious health risks and undermine patient confidence in the service. The clinical audit and interventions implemented helped to reduce errors in medication recording in discharge summaries.
\end{abstract}

Declaration of interest None.
Accurate communication between primary care and hospital is essential to the continuity of patient care. Despite considerable National Health Service (NHS) investment in information technology in recent years, paper discharge summaries remain the principal method of communication between most general practitioners (GPs) and hospitals. Discharge summaries have been shown to contain various types of discrepancies, which have been linked with a number of possible risks to patients, including medication errors and adverse drug events. ${ }^{1,2}$

The National Institute for Health and Clinical Excellence (NICE) has produced relevant guidance on medicine reconciliation on discharge ${ }^{3}$ and supporting medicine adherence. ${ }^{4}$ Electronic systems may replace written summaries in the longer term. Studies of electronic prescribing systems have shown however that there are complex interactions between these and their users, and that this can lead to further errors where there is inadequate testing, training and evaluation of such systems. ${ }^{5}$

We used a completed cycle of clinical audit to improve the accuracy of medication information provided in discharge summaries at the Caludon Centre, an in-patient psychiatric unit in Coventry, UK.

\section{Method}

The audit's objectives were to improve the recording of in-patient medication in discharge summaries and to ensure GPs were advised on how long to continue as required
(PRN) sedative medications. We reviewed discharge summaries of 100 patients consecutively discharged from the general adult wards in the last 5 months of 2004. Results were analysed in SPSS version 12 for Windows. Admissions without any in-patient prescription and those without discharge summaries were discounted from the final analysis. Results were then fed back at a postgraduate educational meeting in 2005 .

Prior to the audit, the to-take-out prescriptions form was A5 size, a triplicate, no-carbon-required set of three sheets, one for pharmacy, one for filing in the records and one for the GP. Due to its small size it was often not easy to read or find in the notes. The GP would usually wait for discharge summaries to be posted or call the centre to clarify the discharge medications.

\section{Recommendations of the first audit}

The following interventions were made.

1 We altered the format of to-take-out prescriptions to a single A4 sheet, adding a section for advice on PRN medications. Ward managers took responsibility to ensure this was faxed to the GP on the day of discharge.

2 A maximum of 2 weeks was allowed for completion of the discharge summary. If the doctor responsible was on leave during this time, the covering doctor was expected to complete the summary.

3 A standard for admission summaries was set; they were to be completed within 7 days of admission. 
4 Medical secretaries had responsibility to keep a chart of timings of admission and discharge summaries. Consultants would supervise discharge summaries more closely and ensure the junior doctors completed the summaries within the time frame.

The changes were presented as a flow chart to the consultants, junior doctors, ward staff and medical secretaries to ensure uniformity. The flow chart was also included in the junior doctors' induction pack. The changes were implemented during the new trainees' induction and reinforced by the consultant audit lead and the junior doctors' representative. Regular meetings were held with junior doctors to remind them of the standards.

\section{Re-audit}

A re-audit was conducted in 2007 looking at 100 consecutive discharges during a 6-month period from the end of 2006. If any notes were missing at the time, the notes of the next patient in the list were taken.

\section{Results}

For both audit cycles we identified 100 discharges within 6 months prior to the audit. We discounted 17 discharges in 2005: 4 were without discharge summaries and 13 without in-patient prescriptions. In 2007 we discounted 14 discharges: 4 were without summaries, 5 were without in-patient prescriptions and 5 individuals were transferred within the trust to be discharged elsewhere. Results for the first objective of the audit, to improve the recording of in-patient medication in discharge summaries, are shown in Table 1.

Results for the second objective, to ensure GPs are advised on PRN sedative medication, are shown in Table 2.

\section{Discussion}

The audit achieved one of its two original objectives. The results suggest the introduction of a guideline for discharge summaries increased the accuracy of medication recording (from 64 to $83 \%$ ) and decreased the number of medications omitted from discharge summaries (from 16 to $8 \%$ ), but it did not alter the frequency of the recording of wrong dosages (7\% in both audits). However, we noticed that at reaudit the mistakes were mostly on physical medications, especially the errors about dosages. A study by Morcos et $a l^{2}$ reported errors in the transfer of medication information at some stage of a completed admission in $91 \%$ of cases reviewed. Our results, although not strictly comparable with that study, confirm the impression that such errors are relatively common in routine clinical practice.

The number of in-patient PRN sedative prescriptions and the frequency of the recording of advice to GPs over these prescriptions both declined sharply (from 18 to $3 \%$ and from 33 to $0 \%$ respectively). It is possible that the first audit highlighted the extent of PRN sedative prescribing and doctors were more vigilant following this. The findings of improvements in record keeping after the introduction of
Table 1 Medication recording in discharge summaries

\begin{tabular}{lcc} 
Audit & $2005, n(\%)^{1}$ & $2007, n(\%)^{1}$ \\
\hline Summaries reviewed & $83(83)$ & $86(86)$ \\
\hline Medications recorded correctly & $53(64)$ & $70(83)$ \\
\hline Missing one or more medications & $13(16)$ & $7(8)$ \\
\hline Wrong dosages recorded & $6(7)$ & $6(7)$ \\
\hline
\end{tabular}

1. $N=100$

Table 2 Advice on as required (PRN) sedative prescribing

\begin{tabular}{lcc} 
Audit & $2005, n(\%)^{1}$ & $2007, n(\%)^{1}$ \\
\hline $\begin{array}{l}\text { In-patients prescribed PRN } \\
\text { sedative }\end{array}$ & $15(18)$ & $3(3)$ \\
\hline $\begin{array}{l}\text { Advice to general practitioner } \\
\text { on continuation at discharge }\end{array}$ & $5(33)$ & $0(0)$
\end{tabular}

1. $N=100$.

written guidance or protocols are consistent with other audits, which have shown similar effects for the completion of risk assessments. ${ }^{6}$

Clinical audit can be used to improve communication between hospitals and GPs. It is, however, labour intensive and sustaining the improvement can prove difficult given constant medical and administrative staff turnover or absences when staff are on leave or off sick. Computergenerated discharge summaries distributed via an online system may provide a more sustainable solution. Pilot studies of such systems have reported positive results, ${ }^{7,8}$ but they still appear a distant prospect for many NHS trusts.

The audit reviewed a limited range of prescribing information recorded in hospital records but did not attempt to investigate the accuracy of these records. Using prescription records meant there was no ambiguity about medication histories. Nevertheless, it is possible that the results are in part the product of variations in the audit samples. For example, more in-patients had no medications prescribed or were prescribed PRN sedatives in the first audit cycle and more patients were subject to transfer from the wards in the second audit. Explanations for these variations are beyond the scope of the audit.

\section{Maintaining improvement and further reducing errors}

Implementing and maintaining change requires careful planning and involvement of different people. As the cohort of junior doctors change every 6 months, regular communication about the importance of ensuring accuracy in medication recording will help to maintain the positive changes. Newer trainees can better meet the standards when these are explained at induction and presented as part of ongoing practice. Errors in dosages can be reduced with closer supervision by consultants and also monitoring by pharmacists, who now regularly attend ward rounds.

Recording medication is an important element of the delivery, sharing and transfer of care. Communication between services must therefore always be of the highest standard. 


\section{Acknowledgements}

We thank Dr Sunil Routhu and Dr Meredith King for assisting with data collection as well as Dr Sadira Teeluckdharry for help in developing the flowchart.

\section{About the authors}

Dr Ashok Kumar Jainer is Consultant in General Adult Psychiatry, Caludon Centre, Coventry and Warwickshire Partnership Trust, Coventry; Dr Fabida Noushad is Specialty Trainee Year 5 (LAT) in General Adult Psychiatry, Bradgate Mental Health Unit, Leicestershire Partnership Trust; Tim Coupe is Clinical Audit Facilitator, Worcestershire Mental Health Partnership NHS Trust, Newtown Hospital, Worcester; Dr Chaya Rekha Mupiri is CT3, Park Royal Hospital, Central and North West London NHS Foundation Trust; Dr Anoop Saraf is Specialty Trainee Year 5, Old Age Psychiatry, South London and Maudsley NHS Foundation Trust.

\section{References}

1 Boockvar KS, Liu S, Goldstein N, Nebeker J, Siu A, Fried T. Prescribing discrepancies likely to cause adverse drug events after patient transfer. Qual Saf Health Care 2009; 18: 32-6.
2 Morcos S, Francis SA, Duggan C. Where are the weakest links? A descriptive study of discrepancies in prescribing between primary and secondary sectors of mental health service provision. Psychiatr Bull 2002; 26: 371-4.

3 National Institute for Health and Clinical Excellence. Technical Patient Safety Solutions for Medicines Reconciliation on Admission of Adults to Hospital. NICE, 2007 (http://guidance.nice.org.uk/PSG001).

4 National Institute for Health and Clinical Excellence. Medicines Adherence: Involving Patients in Decisions about Prescribed Medicines and Supporting Adherence. NICE, 2009 (http://guidance.nice.org.uk/ CG76).

5 Miller RA, Gardner RM, Johnson KB, Hripcsak G. Clinical decision support and electronic prescribing systems: a time for responsible thought and action. J Am Med Inform Assoc 2005; 12: 403-9.

6 Masson N, Liew A, Taylor J, McGuigan F. Risk assessment of psychiatric in-patients: audit of completion of a risk assessment tool. Psychiatr Bull 2008; 32: 13-4.

7 Kirby J, Barker B, Fernando D, Jose M, Curtis C, Goodchild A, et al. A prospective case control study of the benefits of electronic discharge summaries. J Telemed Telecare 2006; 12: 20-1.

8 Scullard $\mathrm{P}$, labal $\mathrm{N}$, White $\mathrm{L}$, Olla $\mathrm{E}$, Thomson GA. Improved communication between hospital and general practice using online patient discharge summaries. J Telemed Telecare 2007; 13: 56-8. 